PROCEEDINGS OF THE

AMERICAN MATHEMATICAL SOCIETY

Volume 140, Number 3, March 2012, Pages 961-969

S 0002-9939(2011)10960-2

Article electronically published on July 18, 2011

\title{
A NOTE ON OPEN 3-MANIFOLDS SUPPORTING FOLIATIONS BY PLANES
}

\author{
CARLOS BIASI AND CARLOS MAQUERA \\ (Communicated by Alexander N. Dranishnikov)
}

\begin{abstract}
We show that if $N$, an open connected $n$-manifold with finitely generated fundamental group, is $C^{2}$ foliated by closed planes, then $\pi_{1}(N)$ is a free group. This implies that if $\pi_{1}(N)$ has an abelian subgroup of rank greater than one, then $\mathcal{F}$ has at least a nonclosed leaf. Next, we show that if $N$ is three dimensional with fundamental group abelian of rank greater than one, then $N$ is homeomorphic to $\mathbb{T}^{2} \times \mathbb{R}$. Furthermore, in this case we give a complete description of the foliation.
\end{abstract}

\section{INTRODUCTION}

In this work we deal with a particular class of codimension one foliations. Two foliated manifolds $\left(N_{1}, \mathcal{F}_{1}\right)$ and $\left(N_{2}, \mathcal{F}_{2}\right)$ are said to be $C^{r}$ conjugate if there exists a $C^{r}$ homeomorphism $h: N_{1} \rightarrow N_{2}$ that takes leaves of $\mathcal{F}_{1}$ onto leaves of $\mathcal{F}_{2}$. Let $\mathcal{F}$ be a $C^{2}$ codimension one foliation defined on an $n$-manifold $N$. A leaf of $\mathcal{F}$ is closed if it is closed as a subset of $N$. If all the leaves are diffeomorphic to $\mathbb{R}^{n-1}$, the foliation $\mathcal{F}$ is called a foliation by planes. The foliation $\mathcal{F}$ is called a Reeb foliation of $N$ if both $N$ and $\mathcal{F}$ are orientable, each leaf of $\mathcal{F}$ in the interior of $N$ is homeomorphic to $\mathbb{R}^{n-1}$, and if $\partial N \neq \emptyset$, then each component of $\partial N$ is a leaf of $\mathcal{F}$ which is homeomorphic to the torus $\mathbb{T}^{n-1}$. This notion was introduced by Rosenberg and Roussarie in [1]. Note that each transversally orientable foliation by planes $\mathcal{F}$ on an orientable open $n$-manifold is a Reeb foliation.

Rosenberg and Roussarie in 11 have proved that the only compact connected 3 -manifolds which admit Reeb foliations are $\mathbb{T}^{3}, D^{2} \times \mathbb{S}^{1}$ and $\mathbb{T}^{2} \times[0,1]$. Novikov 8] has proved that all Reeb foliations of $D^{2} \times \mathbb{S}^{1}$ are topologically equivalent to the Reeb component. Chatelet and Rosenberg 2 and Rosenberg and Roussarie 10. have classified Reeb foliations of class $C^{2}$ of $\mathbb{T}^{2} \times[0,1]$ and $\mathbb{T}^{3}$, respectively. In both cases, the authors prove, among other things, that all the leaves of $\mathcal{F}$ are dense (in particular there is no closed leaf). Let us observe that in these two cases the fundamental group of $N$ is abelian of rank greater than one. In higher

Received by the editors June 15, 2009 and, in revised form, May 28, 2010; August 28, 2010; and December 18, 2010.

2010 Mathematics Subject Classification. Primary 37C85; Secondary 57R30.

Key words and phrases. Foliation by planes, open manifolds, incompressible torus, fundamental group, free group.

The first author was supported by FAPESP Grant 2008/57607-6.

The second author was supported by CNPq and FAPESP Grants 2008/57607-6 and 2009/17493-4. 
dimension, that is, when $N$ is closed and $n$-dimensional, Rosenberg 13 proved that $\pi_{1}(N)$ is free abelian. Recently, Álvarez López, Arraut and Biasi [1] proved that if $N \backslash\left\{p_{1}, \ldots, p_{k}\right\}$ is foliated by closed planes, then $N=S^{n}$ (the $n$-sphere) and $k=1$.

Palmeira 9] studied transversally orientable $C^{2}$ foliations by closed planes on open orientable $n$-manifolds $N$, which have finitely generated fundamental group, and proved that these foliations are $C^{2}$ conjugated to the product of a foliation on an open surface by $\mathbb{R}^{n-2}$. This implies that the fundamental group of $N$ is free. Remember that any free group is the free product $H_{1} * \cdots * H_{\ell}$ where $H_{i}, 1 \leq i \leq l$, is isomorphic to $\mathbb{Z}$.

In this paper we consider foliations by planes on an open and connected $n$ manifold $N$. We try to initiate the classification of some $C^{2}$ foliations by planes in open manifolds having at least one leaf not closed.

Our first result is the following.

Theorem 1.1. Let $N$ be an open connected $n$-manifold with a $C^{2}$ foliation by closed planes. If $\pi_{1}(N)$ is finitely generated, then $\pi_{1}(N)$ is free.

The difference from Palmeira's result is that it is not necessary to assume that $N$ is orientable and that the foliation is transversally orientable. As a consequence of Theorem 1.1, we have

Corollary 1.2. Let $N$ be an open connected $n$-manifold with a $C^{2}$ foliation by planes. If $\pi_{1}(N)$ has an abelian subgroup of rank greater than one, then $\mathcal{F}$ has at least a leaf which is not closed.

Obviously, the reciprocal of this result is not true; see (2) of Example 2.1. However, the previous corollary motivates the following natural question: "If $\pi_{1}(N)$ has an abelian subgroup (or is abelian) of rank greater than one, then what can we say about the set of nonclosed leaves?" In this direction, when $N$ is three dimensional, we obtain the following result:

Theorem 1.3. Let $N$ be an open connected orientable 3 -manifold with a $C^{2}$ foliation by planes $\mathcal{F}$ and assume that $\pi_{1}(N)$ is finitely generated and is a free product of abelian subgroups. Then

(1) $\pi_{1}(N)=H_{1} * \cdots * H_{\ell}$, where $H_{j}, j=1, \ldots, \ell$, is a subgroup of $\pi_{1}(N)$ isomorphic to $\mathbb{Z}$ or $\mathbb{Z}^{2}$;

(2) for each $j=1, \ldots, \ell$, satisfying that $H_{j}$ is isomorphic to $\mathbb{Z}^{2}$, there exists an open submanifold $N_{j}$ of $N$ that is invariant by $\mathcal{F}$, such that $\pi_{1}\left(N_{j}\right)=H_{j}$ and $\left(\left.\mathcal{F}\right|_{N_{j}}, N_{j}\right)$ is topologically conjugated to $\left(\mathcal{F}_{0} \times \mathbb{R}, \mathbb{T}^{2} \times \mathbb{R}\right)$, where $\mathcal{F}_{0}$ is the foliation on the 2-torus $\mathbb{T}^{2}$ which is defined by the irrational flow.

By using this theorem we obtain

Theorem 1.4. Let $N$ be an open connected orientable 3-manifold with a $C^{2}$ foliation by planes $\mathcal{F}$ and assume that $\pi_{1}(N)$ is finitely generated. If $\pi_{1}(N)$ is an abelian group of rank two, then $N$ is homeomorphic to $\mathbb{T}^{2} \times \mathbb{R}$. Moreover, there exists an open submanifold $N_{0}$ which is homeomorphic to $\mathbb{T}^{2} \times \mathbb{R}$ and invariant by $\mathcal{F}$ such that

(1) $\left(\left.\mathcal{F}\right|_{N_{0}}, N_{0}\right)$ is topologically conjugated to $\left(\mathcal{F}_{0} \times \mathbb{R}, \mathbb{T}^{2} \times \mathbb{R}\right)$, where $\mathcal{F}_{0}$ is the foliation on the 2-torus defined by the irrational flow;

(2) every connected component $B$ of $N \backslash \overline{N_{0}}$ is foliated by closed planes in $B$, hence, homeomorphic to $\mathbb{R}^{3}$. Furthermore, each leaf $L$ in $B$ separates $N$ in two connected components. 
We observe that in the hypothesis of Theorem 1.4. by Proposition 4.3, we may assume that $\pi_{1}(N)$ has an abelian subgroup of rank greater than one.

The proof Theorem 1.3 is particular to the three-dimensional case, since we use a result of 4 on the existence of an incompressible torus in irreducible 3-manifolds.

This paper is organized as follows. In Section 2 we present some examples of foliations by planes in open manifolds having leaves not closed. In Section 3, by using two remarkable results obtained by Palmeira (Theorem 3.1) and Stallings (Lemma 3.4), we prove Theorem 1.1. In Section 4 we prove Theorem 1.3 , and then by using this theorem we show Theorem 1.4

\section{EXAMPLES}

Let us give some examples of open manifolds supporting foliations by planes having leaves that are not closed.

Example 2.1. Let $X$ be the vector field on the 2-torus $\mathbb{T}^{2}$ which is defined by the irrational flow. Fix a point $p$ in $\mathbb{T}^{2}$ and let $f: \mathbb{T}^{2} \rightarrow \mathbb{R}$ be an application such that $f(p)=0$ and $f(x) \neq 0$ for all $x \in \mathbb{T}^{2}-\{p\}$. Let $\mathcal{F}_{0}$ be the one-dimensional foliation in $\mathbb{T}^{2}-\{p\}$ which is defined by the vector field $f X$. We obtain two foliations:

(1) the foliation by planes $\mathcal{F}_{1}$ on $N=\mathbb{T}^{2} \times \mathbb{R}^{n-2}$ whose leaves are the product of the orbits of $X$ with $\mathbb{R}^{n-2}$;

(2) the foliation by planes $\mathcal{F}_{2}$ on $N=\left(\mathbb{T}^{2}-\{p\}\right) \times \mathbb{R}^{n-2}$, whose leaves are the product of leaves of $\mathcal{F}_{0}$ with $\mathbb{R}^{n-2}$.

We can observe that every leaf in the two foliations $\mathcal{F}_{1}$ and $\mathcal{F}_{2}$ is dense, in particular, not closed. But, in the first case the fundamental group of $N$ is isomorphic to $\mathbb{Z}^{2}$, and in the second case the fundamental group of $N$ is free and not abelian with two generators (because $\pi_{1}(N)=\pi_{1}\left(\mathbb{T}^{2}-\{p\}\right)$ ).

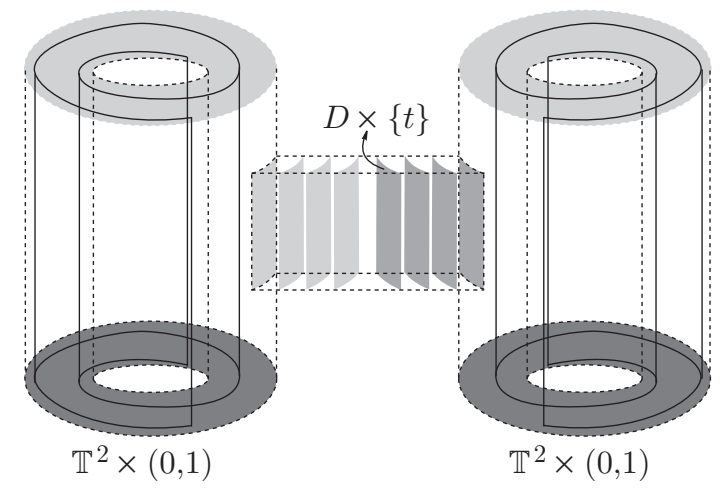

Figure 1. Example 2.1

Example 2.2. In [2, page 96] the authors defined a $C^{\infty}$ locally free action of $\mathbb{R}^{2}$ on $\mathbb{T}^{2} \times[0,1]$ such that $T_{0}=\mathbb{T}^{2} \times\{0\}$ and $T_{1}=\mathbb{T}^{2} \times\{1\}$ are the only compact orbits and every orbit in $\mathbb{T}^{2} \times(0,1)$ is a dense plane. Let $\mathcal{F}_{0}$ be the $C^{\infty}$ foliation on $\mathbb{T}^{2} \times[0,1]$ defined by the orbits of this foliation. Let $D$ be an open 2-disk in $T_{1}$. We consider in $D \times[0,1]$ the foliation $\mathcal{F}_{d}$ whose leaves are $D \times\{t\}$ with $t \in[0,1]$. Let $N$ be the open 3 -manifold which is obtained by gluing two copies of 
$\left[\mathbb{T}^{2} \times(0,1)\right] \cup D$ with $D \times I$ as in Figure 1 Let $\mathcal{F}$ be the foliation in $N$ such that $\left.\mathcal{F}\right|_{\mathbb{T}^{2} \times(0,1)}=\left.\mathcal{F}_{0}\right|_{\mathbb{T}^{2} \times(0,1)}$ and $\left.\mathcal{F}\right|_{D \times I}=\mathcal{F}_{d}$. Then we have the following facts:

(1) The fundamental group of $N$ is the free product $\mathbb{Z}^{2} * \mathbb{Z}^{2}$.

(2) By $(2.5)$ of 3 , in each copy of $\mathbb{T}^{2} \times(0,1)$ we can find two incompressible 2 -tori $T_{1}$ and $T_{2}$, which are transverse to $\mathcal{F}$, such that the foliations induced by $\mathcal{F}$ in these tori are topologically equivalent to the irrational flow on the 2 -torus.

(3) for $i=1,2$, let $\operatorname{Sat}\left(T_{i}\right)$ be saturated by $\mathcal{F}$ of $T_{i}$. Then, the set $\operatorname{Sat}\left(T_{i}\right)$ is equal to $\mathbb{T}^{2} \times(0,1)$ and the complement of the closure of $\operatorname{Sat}\left(T_{1}\right) \cup \operatorname{Sat}\left(T_{2}\right)$ is $D \times(0,1)$ which is foliated by closed planes.

Example 2.3. For $k \geq 2$, let $\mathcal{F}_{0}$ be a $(k-1)$-dimensional foliation by planes of class $C^{2}$ on the $k$-torus $\mathbb{T}^{k}$. In $N=\mathbb{T}^{k} \times \mathbb{R}^{n-k}$, we consider the foliation by planes $\mathcal{F}$ which is the product of $\mathcal{F}_{0}$ with $\mathbb{R}^{n-k}$. Then:

(1) $\pi_{1}(N)=\pi_{1}\left(\mathbb{T}^{k}\right)=\mathbb{Z}^{k}$ (in particular, free abelian).

(2) All the leaves of $\mathcal{F}$ are dense (in particular, they are not closed). This occurs because every leaf of $\mathcal{F}_{0}$ is dense in $\mathbb{T}^{k}$.

(3) $N$ is not homeomorphic to the product of a surface by $\mathbb{R}^{n-2}$.

(4) $\operatorname{rank}\left(\pi_{1}(N)\right)=k \geq 2$.

\section{THE FUNDAMENTAL GROUP OF AN OPEN $n$-MANIFOLD FOLIATED BY CLOSED PLANES}

3.1. Proof of Theorem 1.1. We state the results to be used in the proof of Theorem 1.1. We start with the following result of Palmeira, which is Corollary 1 of Section 5 in 9 .

Theorem 3.1. If $N$ is an orientable open $n$-manifold, $n \geq 3$, which has a finitely generated fundamental group and with a transversely orientable $C^{2}$ foliation $\mathcal{F}$ by closed planes, then there exists an orientable surface $\Sigma$ and an orientable onedimensional foliation $\mathcal{F}_{0}$ of $\Sigma$ such that $(N, \mathcal{F})$ is conjugated by a diffeomorphism to $\left(\Sigma \times \mathbb{R}^{n-2}, \mathcal{F}_{0} \times \mathbb{R}^{n-2}\right)$.

Note that since the foliation $\mathcal{F}_{0}$ is by closed lines, the surface $\Sigma$ is not a closed surface.

Corollary 3.2. $N$ has the same homotopy type of an open surface.

It is well known that the existence of a nonclosed leaf of $\mathcal{F}$ implies the existence of a simple closed curve $\gamma$ transversal to $\mathcal{F}$. Moreover, by using Heafliger's arguments, we have that $\gamma$ is necessarily not nullhomotopic. Consequently:

Corollary 3.3. In Theorem 3.1, when $N$ is simply connected, it is not necessary to assume either that $\mathcal{F}$ is transversely orientable or that the leaves are closed, and moreover $\Sigma=\mathbb{R}^{2}$ in this case.

Theorem 3.1 and the following result, which was conjectured by Serre 14 and proved by Stallings [15, are crucial to proving Theorem 1.1

Lemma 3.4 ([15], Stallings). Let $G$ be a torsion-free finitely generated group. If $H$ is a free subgroup of finite index, then $G$ is also free.

Let $X$ be a topological space and $G$ a group. We say that $X$ is a $K(G, 1)$-space if $\pi_{1}(X)=G$ and $\pi_{i}(X)=\{1\}, i \geq 2$. 
Proposition 3.5. If $N$ is an open connected $n$-manifold foliated by planes, then $\pi_{1}(N)$ is torsion-free. In particular, if $\pi_{1}(N)$ is finite, then $N$ is diffeomorphic to $\mathbb{R}^{n}$.

Proof. We first claim that $N$ is a $K\left(\pi_{1}(N), 1\right)$-space. In fact, let $\widetilde{N}$ be the universal covering of $N$ and let $\widetilde{\mathcal{F}}$ be the lifting on $\widetilde{N}$ of $\mathcal{F}$. It is well known that for manifolds $\pi_{i}(N)=\pi_{i}(\widetilde{N})$ for all $i \geq 2$. Applying Corollary 3.3 for $\widetilde{N}$ and $\widetilde{\mathcal{F}}$, we have that $\widetilde{N}=\mathbb{R}^{n}$. Thus, $\pi_{i}(\tilde{N})=\{1\}$, for all $i=1,2, \ldots, n$. This concludes the proof of our claim.

Finally, if $\pi_{1}(N)$ has nontrivial torsion, then, by Lemma 9.4 of [7, any $K\left(\pi_{1}(N)\right.$, 1) space has infinite dimension, in particular $N$. This contradiction proves that $\pi_{1}(N)$ is torsion-free. Furthermore, if $\pi_{1}(N)$ is finite, we obtain that $\pi_{1}(N)$ is trivial; hence by Corollary 3.3 we have that $N$ is diffeomorphic to $\mathbb{R}^{n}$.

Proof of Theorem 1.1. We have the following possibilities:

(a) $N$ is orientable and $\mathcal{F}$ transversally orientable: by Palmeiras' theorem, $\pi_{1}(N)$ is free.

(b) $N$ is not orientable or $\mathcal{F}$ is not transversally orientable: we consider $\pi$ : $\widetilde{N} \rightarrow N$ to be the covering map of degree at most 4 that turn $\widetilde{N}$ orientable and $\widetilde{\mathcal{F}}$, the lifting to $\widetilde{N}$ of $\mathcal{F}$, transversally orientable. Then, by item (a) and Proposition 3.5. we have that $\pi_{1}(\widetilde{N})$ is free and torsion-free, respectively. Finally, since the index of $\pi_{*}\left(\pi_{1}(\widetilde{N})\right)$ in $\pi_{1}(N)$ is at most 4 , it follows from Lemma 3.4 that $\pi_{1}(N)$ is free.

Let $X$ be a finite set and denote by $\# X$ the cardinality of $X$. Let $G$ be a torsion-free finitely generated group. The rank of $G$ is given by

$$
\operatorname{rank}(G)=\min \{k \in \mathbb{N} ; k=\# X \text { where } G \text { is generated by } X\} .
$$

The following result is equivalent to Corollary 1.2 .

Theorem 3.6. Let $\mathcal{F}$ be a foliation by planes of an open connected n-manifold $N$. If every leaf of $\mathcal{F}$ is closed, then each finitely generated abelian subgroup $H$ of $\pi_{1}(N)$ has rank at most one.

Proof. Let $p: \widetilde{N} \rightarrow N$ be the covering map associated to $H$; that is, $\pi_{1}(\widetilde{N})=H$. Then $\widetilde{\mathcal{F}}$, the lifting to $\widetilde{N}$ of $\mathcal{F}$, is a foliation by closed planes. Hence, by Theorem 1.1 $\pi_{1}(\widetilde{N})$ is a free group. Consequently, since $H=\pi_{1}(\widetilde{N})$ is an abelian group, we have that $H$ is equal to $\{1\}$ or is isomorphic to $\mathbb{Z}$. This completes the proof.

Corollary 3.7. Let $\mathcal{F}$ be a foliation by planes of an open connected $n$-manifold $N$, which has a finitely generated fundamental group. If each leaf is closed and $\pi_{1}(N)$ is not abelian, then $C=Z\left(\pi_{1}(N)\right)$, the center of $\pi_{1}(N)$, is trivial.

Proof. Suppose by contradiction that $C \neq\{1\}$. It follows from Theorem 3.6 that $C$ has rank one; hence $C$ is generated by some $\beta$ in $\pi_{1}(N)$. Moreover, the rank of the fundamental group $\pi_{1}(N)$ is at least two; otherwise $\pi_{1}(N)$ is abelian (in that case $C$ would be equal to $\pi_{1}(N)$ ), contrary to the hypothesis. Hence, since $\pi_{1}(N)$ is free (Theorem 1.1), if $E$ is a set of free generators of $\pi_{1}(N)$, then there are two different elements $e_{1}$ and $e_{2}$ in $E$. 
We consider $H_{1}$ and $H_{2}$, the subgroups of $\pi_{1}(N)$ generated by $\left\{e_{1}, \beta\right\}$ and $\left\{e_{2}, \beta\right\}$, respectively. Clearly these subgroups are abelian and, by Theorem 3.6. both have rank one. Suppose that $c_{1}$ (resp. $c_{2}$ ) is a generator of $H_{1}\left(\right.$ resp. $\left.H_{2}\right)$. Then, there are integers $m_{1}, m_{2}, p_{1}, p_{2}$ so that $\beta=c_{i}^{m_{i}}$ and $e_{i}=c_{i}^{p_{i}}$ for $i=1,2$. Hence, we obtain $e_{1}^{m_{1}}=\beta^{p_{1}}$ and $e_{2}^{m_{2}}=\beta^{p_{2}}$. This implies that $e_{1}^{m_{1} p_{2}}=e_{2}^{m_{2} p_{1}}$. This contradicts the fact that $E$ is a set of free generators of $\pi_{1}(N)$ and finishes the proof.

Remark 3.8. Note that if $\pi_{1}(N)$ finitely generated has an abelian subgroup of rank greater than one, or $C \neq\{1\}$, then $N$ cannot be foliated by closed planes.

\section{Classifying open 3-Manifolds foliated By Planes Whose FUNDAMENTAL GROUP IS ABELIAN OF RANK AT LEAST TWO}

In this section we prove Theorems 1.3 and 1.4. We state some results about 3 -manifold topology and foliations by planes which will be used in the proof of Theorems 1.3 and 1.4. We begin with some terminology. A 3-manifold $N$ is called irreducible if every embedded 2 -sphere in $N$ bounds a 3 -ball in $N$. A two-sided surface $S$ in $N$ is incompressible if the map $\pi_{1}(S) \rightarrow \pi_{1}(N)$ induced by the inclusion is injective. The following results are well-know tools in 3-manifold topology.

Theorem 4.1 ([12, Theorem 6]). Let $N$ be an orientable 3 -manifold, not necessarily compact, and let $\mathcal{F}$ be a foliation of $N$ by planes. Then $N$ is irreducible.

Gabai, in [4, Proof of Corollary 8.6], proved the following result.

Theorem 4.2. If $N$ is an open orientable irreducible 3 -manifold and $\mathbb{Z} \times \mathbb{Z}$ is a subgroup of $\pi_{1}(N)$, then $N$ contains an incompressible torus.

4.1. Proof of Theorem 1.3. The following result is crucial for the proof of Theorem 1.3 ,

Proposition 4.3. Let $\mathcal{F}$ be a $C^{2}$ foliation by planes of an open connected $n$ manifold $N$. If $H \cong \mathbb{Z}^{k}$ is a subgroup of $\pi_{1}(N)$, then $k<n$.

To prove this proposition, we need the following classical result in algebraic topology; see for example [6].

Lemma 4.4. Let $X$ be a normal space, $F$ a closed subspace of $X$, and $Y$ a Euclidean neighborhood retract (ENR). Suppose that $f_{0}: F \rightarrow Y$ and $g: X \rightarrow Y$ are continuous maps. If $f_{0}$ is homotopic to $g_{0}=\left.g\right|_{F}$, then there exists a continuous map $f: X \rightarrow Y$ such that $\left.f\right|_{F}=f_{0}$ and $f$ is homotopic to $g$.

Proof of Proposition 4.3. In the proof of Proposition 3.5 we have shown that $N$ is a $K\left(\pi_{1}(N), 1\right)$-space. We have two possibilities:

(a) Case $H=\pi_{1}(N)$. By contradiction, we suppose that $\pi_{1}(N) \cong \mathbb{Z}^{n}$. Since $N$ and $\mathbb{T}^{n}$ are $K\left(\mathbb{Z}^{n}, 1\right)$-spaces, we obtains that $N$ and $\mathbb{T}^{n}$ have the same homotopic type. Consequently, by a classical theorem of algebraic topology (Whitehead's theorem), $H_{p}(N)=H_{p}\left(\mathbb{T}^{n}\right)$ for all $p=1, \ldots, n$. But, as $N$ is open and connected, $H_{n}(N)=0$. This contradicts the fact that $H_{n}(N)=H_{n}\left(\mathbb{T}^{n}\right)=\mathbb{Z}$ and therefore proves that $k<n$. 
(b) General case. Let us consider $p: \widehat{N} \rightarrow N$ to be the covering map associated to $H$; that is, $\pi_{1}(\widehat{N})=H$. Let $\widehat{\mathcal{F}}$ be the foliations of $\widehat{N}$ induced by $\mathcal{F}$; this foliation is by planes. Thus, by the first case we obtain $k<n$.

Proof of Theorem 1.3. By Proposition 4.3, every abelian subgroup of $\pi_{1}(N)$ is isomorphic to $\mathbb{Z}$ or to $\mathbb{Z}^{2}$. Consequently, because $\pi_{1}(N)$ is a free product of abelian groups, the fundamental group of $N$ is a free product of the form

$$
\pi_{1}(N)=H_{1} * \cdots * H_{\ell},
$$

where $H_{j}, j=1, \ldots, \ell$, are subgroups of $\pi_{1}(N)$ isomorphic to $\mathbb{Z}$ or $\mathbb{Z}^{2}$, respectively.

We suppose that $j \in\{1, \ldots, \ell\}$ is such that $H_{j}$ is isomorphic to $\mathbb{Z}^{2}$. By Theorems 4.1 and 4.2 there exists an incompressible 2-torus $T_{j}$ contained in $N$ such that $\pi_{1}\left(T_{j}\right)=H_{j}$. Deforming $T_{j}$, if necessary, by an isotopy of identity, we can assume that $T_{j}$ is a $C^{2}$ embedding which is in general position with respect to $\mathcal{F}$. Then, the $C^{2}$ foliation $\mathcal{F}$ induces a $C^{2}$ foliation $\mathcal{G}_{j}$ on $T_{j}$ having finitely many singularities each of which is locally topologically equivalent either to a center or to a saddle point of a vector field. We can assume that the foliation $\mathcal{G}_{j}$ is defined by a $C^{2}$ vector field $G_{j}$. Certainly, we may assume that $T_{j}$ has been chosen so that no pair of singularities of $G_{j}$ is in the same leaf of $\mathcal{F}$; in other words, $G_{j}$ has no saddle connections.

(a) $G_{j}$ is topologically equivalent to irrational flow.

In fact, by using Rosenberg's arguments (see [12, pag. 137]), we may deform $T_{i}$ by a $C^{2}$ isotopy of identity so that $G_{j}$ has no singularities. Hence, since $\mathcal{F}$ is by planes and $T_{j}$ is incompressible, $G_{j}$ has no closed orbit. Therefore, as the vector field $G_{j}$ is of class $C^{2}$, we have that $G_{j}$ is topologically equivalent to irrational flow.

Let $N_{j}$ be saturated by $\mathcal{F}$ of $T_{j}$. Clearly, $N_{j}$ is an open 3-submanifold of $N$ invariant by $\mathcal{F}$. We will show that $N_{j}$ is homeomorphic to $\mathbb{T}^{2} \times \mathbb{R}$. Let $\mathcal{F}_{j}$ be the restriction of $\mathcal{F}$ to $N_{j}$ and let $p: T \mathcal{F}_{j} \rightarrow N_{j}$ be the vector bundle such that for all $x \in N_{j}$ the fiber $p^{-1}(x)$ is the tangent at $x$ of the leaf of $\mathcal{F}_{j}$, which is also a leaf of $\mathcal{F}$. Let $\theta$ be the normal vector field to $T_{j}$ in $N_{j}$ and let $\left.T \mathcal{F}_{j}\right|_{T_{j}}$ be the restriction of $T \mathcal{F}_{j}$ to $T_{j}$. By deforming $T_{j}$, if necessary, we can assume that:

(b) $\theta$ is tangent to $\mathcal{F}$; that is, $\theta:\left.T_{j} \rightarrow T \mathcal{F}_{j}\right|_{T_{j}}$.

By the proof of Proposition 3.5, we have that $N_{j}$ is a $K\left(\pi_{1}\left(N_{j}\right), 1\right)$-space. Then, by Whitehead's theorem, the inclusion $i: T_{j} \rightarrow N_{j}$ is a homotopy equivalence. Consequently:

(c) there exists a continuous map $f: N_{j} \rightarrow T_{j}$ such that $f \circ i$ is homotopic to $i d_{T_{j}}$ and $i \circ f$ is homotopic to $i d_{N_{j}}$.

We claim that

(d) there exists a retraction $r: N_{j} \rightarrow T_{j}$ such that $\left.r\right|_{T_{j}}=i d_{T_{j}}$ and $r$ is homotopic to $f$.

Indeed, calling $X=N_{j}, Y=F=T_{j}, f_{0}=i d_{T_{j}}: T_{j} \rightarrow T_{j}$ and $g=f: N_{j} \rightarrow T_{j}$, by Lemma 4.4, there exists $r: N_{j} \rightarrow T_{j}$ such that $\left.r\right|_{T_{j}}=i d_{T_{j}}$ and $r$ is homotopic to $f$.

(e) $N_{j}$ is homeomorphic to $\mathbb{T}^{2} \times \mathbb{R}$.

It follows from (c) and (d) that $i \circ r: N_{i} \rightarrow N_{j}$ is homotopic to $i d_{N_{j}}$. Hence, by a classical result (see for example Theorem 11.5 of [16]) we have that the vector bundles $(i \circ r)^{*}\left(T \mathcal{F}_{j}\right)$ and $T \mathcal{F}_{j}$ are equivalent. On the other hand, since $i \circ r=r$, 
we obtain that $(i \circ r)^{*}\left(T \mathcal{F}_{j}\right)=r^{*}\left(\left.T \mathcal{F}_{j}\right|_{T_{j}}\right)$. Consequently, the vector field $\Theta=r^{*} \theta$ is tangent to $\mathcal{F}_{j}$ and is normal to $T_{j}$. This implies that $N_{j}$ is homeomorphic to $\mathbb{T}^{2} \times \mathbb{R}$.

Finally, if for $i \neq j$ the group $H_{i}$ is isomorphic to $\mathbb{Z}^{2}$, then we obtain another open 3-submanifold of $N$ which is invariant by $\mathcal{F}$ and homeomorphic to $\mathbb{T}^{2} \times \mathbb{R}$. Furthermore, $N_{i} \cap N_{j}=\emptyset$.

\subsection{Proof of Theorem $\mathbf{1 . 4}$}

Proof of Theorem 1.4. By Theorem 1.3, there exists $N_{0}$ an open submanifold of $N$ homeomorphic to $\mathbb{T}^{2} \times \mathbb{R}$ which is invariant by $\mathcal{F}$ such that $\left(\left.\mathcal{F}\right|_{N_{0}}, N_{0}\right)$ is topologically equivalent to $\left(\mathcal{F}_{0} \times \mathbb{R}, \mathbb{T}^{2} \times \mathbb{R}\right)$ where $\mathcal{F}_{0}$ is the foliation on $\mathbb{T}^{2}$ defined by the irrational flow. Note that $\pi_{1}\left(N_{0}\right)=\pi_{1}(N) \cong \mathbb{Z}^{2}$.

Let $B$ be a connected component of $N \backslash \overline{N_{0}}$. Then

(a) $B$ is homeomorphic to $\mathbb{R}^{3}$ and every leaf in $B$ is closed.

First of all we claim that $\pi_{1}(B)=\{1\}$. In fact, by using the Van Kampen theorem, we have that $\pi_{1}(B) * \pi_{1}\left(N_{0}\right)$ is a subgroup of $\pi_{1}(N)$ and hence, since $\pi_{1}\left(N_{0}\right)=\pi_{1}(N)$, we have that $\pi_{1}(B)=\{1\}$. Now, we are going to show that every leaf in $B$ is closed in $B$. We suppose, by contradiction, that there exists a leaf in $B$ which is not closed in $B$. By classical arguments in the theory of foliations, there exists a simple closed curve $\gamma \subset B$ which is transversal to $\mathcal{F}$. Moreover, $\gamma$ is not nullhomotopic in $B$; otherwise, by using Heafliger's arguments, we obtain a loop $\alpha$ in a leaf of $\mathcal{F}$ which is nontrivial in this leaf, contradicting the fact that all the leaves are planes. Therefore $\gamma$ is not nullhomotopic in $B$, but this contradicts the fact that $\pi_{1}(B)=\{1\}$. Thus every leaf in $B$ is closed. Thence, by Palmeira's theorem, we have that $B$ is homeomorphic to $\mathbb{R}^{3}$ and $\left(\left.\mathcal{F}\right|_{B}, B\right)$ is topologically equivalent to $\left(\mathcal{F}_{b} \times \mathbb{R}, \mathbb{R}^{2} \times \mathbb{R}\right)$ where $\mathcal{F}_{b}$ is an foliation by lines on $\mathbb{R}^{2}$.

Finally by using the Van Kampen theorem it follows that every leaf in $B$ separates $N$.

\section{REFERENCES}

1. J. A. Álvarez López, J. L. Arraut and C. Biasi, Foliations by planes and Lie group actions. Ann. Pol. Math. 82 (2003), 61-69. MR2041398 (2004m:57071)

2. G. Chatelet and H. Rosenberg, Un théorème de conjugaison des feuilletages. Ann. Inst. Fourier (Grenoble) 213 (1971), 95-106. MR0346807 (49:11531)

3. G. Chatelet, H. Rosenberg and D. Weil, A classification of the topological types of $\mathbb{R}^{2}$ actions on closed orientable 3-manifolds. Pub. Math. IHES 43 (1974), 261-272. MR0346809 $(49: 11533)$

4. David Gabai, Convergence groups are Fuchsian groups. Ann. of Math. (2) 136 (1992), 447510. MR:1189862(93m:20065)

5. C. Godbillon, Feuilletages Études géométriques. Progress in Mathematics, Birkhäuser (1991). MR.1120547 (93i:57038)

6. A. Hatcher, Algebraic Topology. Cambridge University Press (2002). MR.1867354 (2002k:55001)

7. J. Hempel, 3-manifolds. Princeton University Press (1976). MR0415619 (54:3702)

8. S. P. Novikov, Topology of foliations. Trudy nzosk. Mm. Obshch. 14 (1970), 513-583. MR 0200938 (34:824)

9. C. F. B. Palmeira, Open manifolds foliated by planes. Ann. of Math. (2) 107 (1978), 109-131. MR0501018 (58:18490)

10. H. Rosenberg and R. Roussarie, Topological equivalence of Reeb foliations. Topology 9 (1970), 231-242. MR0263107 (41:7712) 
11. H. Rosenberg and R. Roussarie, Reeb foliations. Ann. of Math. (2) 91 (1970), 1-24. MR0258057 (41:2704)

12. Harold Rosenberg, Foliations by planes. Topology 7 (1968), 131-138. MR0228011 (37:3595)

13. Harold Rosenberg, Actions of $\mathbb{R}^{n}$ on manifolds. Comment. Math. Helvet. (1966), 36-44. MR 0206978 (34:6794)

14. J-P. Serre, Sur la dimension cohomologique des grupes profinis. Topology 3 (1965), 413-420. MR 0180619 (31:4853)

15. John R. Stallings, On torsion-free groups with infinitely many ends. Ann. of Math. (2) 88 (1968), 312-334. MR0228573 (37:4153)

16. Norman Steenrod, The Topology of Fibre Bundles. Princeton Landmarks in Math., Princeton Univ. Press (1951). MR0039258 (12:522b)

Departamento de Matemática, Instituto de Ciências Matemáticas e de ComputaÇão, Universidade de São Paulo-São Carlos, Av. do Trabalhador São-Carlense 400, 13560970 SÃo Carlos, SP, Brazil

E-mail address: biasi@icmc.usp.br

Departamento de Matemática, Instituto de Ciências Matemáticas e de Computação, Universidade de São Paulo-São Carlos, Av. do Trabalhador São-Carlense 400, 13560970 SÃo CARLos, SP, BRAZIL

E-mail address: cmaquera@icmc.usp.br 